Der dritte Term ist:

$$
-0.003 \sin (\theta-2 \odot)=+0.003 \sin (\odot-\tau)=+0.003 \cos \left(\odot-255^{\circ}\right) \text {. }
$$

Wenn man diesen Term mit dem ersten kombiniert, findet man:

$$
+ \text { o."or } 2 \cos \left(\odot-278^{\circ}\right) \text {. }
$$

Von den weiteren Termen ist keiner größer als ०."०० $r$.

Dr. Chandler hat in A. J. $53^{\circ}$ den Einfluß der Stern. parallaxe auf die Breitenvariation nachgewiesen. Wenn man

für die mittlere Parailaxe der benutzten Sterne o".or 7 annimmt, findet man $+0 ., 005 \cos \left(\odot-270^{\circ}\right)$. Kombiniert man dies mit +0 ". $22 \cos \left(\odot-278^{\circ}\right)$, so erhält man

$$
+0.017 \cos \left(\odot-276^{\circ}\right) \text {. }
$$

Das ist schon ungefähr die Hälfte des KimuraPhänomens.

Groningen, September 1904.

W. de Sitter.

\title{
Beobachtungen von Kometen und Planeten
}

am 6zölligen Steinheilschen Refraktor der Sternwarte des k. u. k. Hydrographischen Amtes in Pola.

\begin{tabular}{l|c|c|c|c|c|c|c|c|}
\hline r 904 & M. Z. Pola & $\Delta \alpha$ & $\Delta \delta$ & Mikr. & $\alpha$ app. & $\log p . \Delta \mid$ & $\delta$ app. & $\log p . \Delta \mid *$ \\
\hline
\end{tabular}

Komet 1904 I.

Mai I $7\left|9^{\mathrm{h}} \mathrm{I} 7^{\mathrm{m}} 4 \mathrm{I}^{\mathrm{s}}\right|+3^{\mathrm{m}} 34^{5} .28 \mid+0^{\prime} 4^{\prime \prime}$. $\mathrm{x}|\mathrm{R}| \mathrm{I} 4^{\mathrm{h}} 57^{\mathrm{m}} 43^{\mathrm{s}} \mathrm{I} 6\left|9.5^{8} 7 \mathrm{n}\right|+57^{\circ} 26^{\prime} 33^{\prime \prime} 2|0.089 \mathrm{n}| \mathrm{I}$

(I) Ceres.

\begin{tabular}{|c|c|c|c|}
\hline \multicolumn{4}{|c|}{ Mittlere Örter der Vergleichsterne. } \\
\hline Gr. & a 1904.0 & 81904.0 & Autorität \\
\hline 8.5 & $6 \div 69$ & $+57^{\circ} 25^{\prime} 49^{\prime \prime} .2$ & 8183 \\
\hline 9.0 & $165^{8} 3$ & $20 \quad 54 \quad 24.9$ & AWe. $13^{\circ} 3^{6}$ \\
\hline .0 & I $6.6 \mathrm{I}$ & $10 \quad 57 \quad 23.2$ & AWe. 12980 \\
\hline
\end{tabular}

\begin{tabular}{rr|rrr|rr|rr|r|rrr|r|rrr|r|r} 
Juni & 4 & I0 & 52 & 19 & -0 & 55.17 & -3 & 9.9 & $R$ & 16 & 57 & 40.59 & $9.149 n$ & -20 & 57 & 29.9 & 0.896 & 2 \\
& 7 & 9 & 43 & 44 & +0 & 29.04 & -6 & 15.0 & $R$ & 16 & 54 & 48.60 & $9.399 n$ & -21 & 3 & 34.2 & 0.882 & 3
\end{tabular}

Beobachter: k. u. k. Linienschiffsfähnrich August Weisbach.

Pola, I 904 Juni 17.

Der Vorstand: Koss, k. u. k. Korv.Kapt.

\section{Ein Fleckenherd auf der Sonne.}

So kann man füglich eine Gegend auf der Sonnenoberfläche bezeichnen, wo in einem verhältnismäßig beschränkten Bezirke im Verlaufe eines Jahres in mindestens acht Rotationen Flecke, teils größere, teils kleinere, teils von kürzerem, teils von längerem Bestande, aufgetreten sind. Es ist die Gegend um den I7o. Meridian der heliographischen Länge (nach Spörers Zählweise) in $12-16^{\circ}$ nördl. Breite. Diese Erscheinung sticht um so mehr hervor, als die gegenwärtige Entwicklung der fleckenbildenden Tätigkeit der Sonne wider Erwarten äußerst langsam vor sich geht, sodaß es, wenn nicht eine plötzliche Steigerung eintritt, den Anschein hat, als ob das kommende Maximum sich beträchtlich verzögerte, oder aber, da $\beta$ es nach Art der Maxima zwischen 1800 und 1830 nur eine mäßige Höhe erreichen werde.

In der bewußten Gegend entstand nach dem Wiedererwachen des Fleckenphänomens das erste mal am 23 . August 1903 in der Nähe des Ostrandes ein Fleckchen, das aber nur zwei Tage dauerte. Am 23. September dagegen entwickelte sich daselbst etwas westlich vom Sonnenzentrum eine Gruppe, die sich in ibrem vorangehenden Teile zu einem schönen behoften Fleck auswuchs und am 29. September im NW unterging. Am I 4 . Oktober erschien sie am Ostrand als isolierter Hoffleck wieder, der bis zum Verschwinden am Nordwestrand in unveränderter Größe verfolgt werden konnte.
Er löste sich dann auf der abgewendeten Seite der Sonne auf; denn bei der vierten Rotation im November war sein Platz leer. Aber im Dezember wat seine Stelle wieder durch einen schönen Hoffleck besetzt, dem im Abstand von $12^{\circ}$ Länge und $6^{\circ}$ nördlich ein gleicher folgte. Nach dem Verschwinden beider Flecke war diese Gegend während der drei nächsten Rotationen fleckenfrei. Gegen Ende März x 904 erwachte dort die Tätigkeit von neuem und dokumentierte sich in einem regelmäßigen Hoffleck. Dieser kehrte bei der nächsten Rotation vergrößert wieder und batte hinter sich eine reich entwickelte Gruppe, die sich taber Io Längengrade erstreckte und mit einem kleineren Hoffleck endigte. Ihre höchste Entfaltung zeigte diese Gruppe am 25. und 26. April, aber danach nahm sie rasch ab und löste sich nach ibrem Untergange am WSW-Rande vollständig auf; denn die nächste Rotation brachte nichts von ihr wieder zum Vorschein. Dagegen enthielt dieselbe Gegend im Juni einen neuen Hoffleck, gefolgt von einigen kleinen Flecken, der vom I3. bis 25. Juni über die Scheibe zog. Ob ex wiedergekehrt ist, kann ich nicht sagen, da ich zu der be. treffenden Zeit ( 10 . bis 22. Juli) nicht beobachtet habe. End lich entstand am 10 . August noch einmal in dieser Gegend ein kleiner Fleck, der sich am 14. August auflöste. Seitdem herrscht hier Ruhe. 